\title{
Surgical resection for patients with recurrent or metastatic gastrointestinal stromal tumors: a protocol for a systematic review and meta- analysis update
}

\author{
Zhaolun Cai ${ }^{1 \dagger}$, Chunyu Xinn ${ }^{2 \dagger}$, Zhiyuan Jiang ${ }^{1 \dagger}$, Chunyu Liu ${ }^{3,4,5,6 \dagger}$, Chaoyong Shen ${ }^{1}$, Guozhen Zhao ${ }^{7}$, Yuan Yin ${ }^{1}$,
} Xiaonan Yin', Zhou Zhao', Mingchun $\mathrm{Mu}^{1}$ and Bo Zhang ${ }^{1,8^{*}}$

\begin{abstract}
Background: There are limited data on the clinical benefits of adding surgical resection in patients with recurrent or metastatic gastrointestinal stromal tumors (GISTs). This protocol outlines the planned scope and methods for a systematic review and meta-analysis update that will compare the clinical outcomes of surgical resection combined with tyrosine kinase inhibitor (TKI) with TKI treatment alone in patients with recurrent or metastatic GISTs.

Methods: This review will update a previously published systematic review by our team. This protocol is presented in accordance with the PRISMA-P guideline. PubMed, Embase, and Cochrane Central Register of Controlled Trials will be systematically searched and supplemented by a secondary screening of the references of all included studies. We will include randomized controlled trials (RCTs) and non-randomized studies (NRS) in this review update. The outcomes evaluated will be overall survival and progression-free survival. Two reviewers will independently screen and select studies, extract data from the included studies, and assess the risk of bias of the included studies. Data extracted from RCTs and NRS will be analysed and reported separately. Preplanned subgroup analyses and sensitivity analyses are detailed within this protocol. The strength of the body of evidence will be assessed using GRADE.
\end{abstract}

Discussion: This systematic review and meta-analysis update will provide a current assessment of the evidence for the role of surgery in patients with recurrent or metastatic advanced GISTs. These findings will be used by the Chinese Society of Clinical Oncology (CSCO) GIST guideline recommendations on surgical treatment for recurrent or metastatic advanced GIST patients in China.

Systematic review registration: This protocol was prospectively registered in the Open Science Framework Registry (https://osf.io/xus7m).

Keywords: Gastrointestinal stromal tumor, Metastatic, Recurrent, Surgery, Tyrosine kinase inhibitor

*Correspondence: hxwcwk@126.com

†Zhaolun Cai, Chunyu Xin, Zhiyuan Jiang and Chunyu Liu contributed equally to this work.

${ }^{8}$ Sanya People's Hospital/West China (Sanya) Hospital, Sichuan University, Sanya 572022, China

Full list of author information is available at the end of the article

\section{Introduction}

Gastrointestinal stromal tumors (GISTs) are the most common mesenchymal tumors of the gastrointestinal tract. GISTs mostly have an activating mutation in the gene encoding the KIT proto-oncogene, receptor tyrosine kinase (KIT) or platelet-derived growth factor receptor alpha (PDGFR $\alpha)$ receptor tyrosine kinase $[1,2]$. original author(s) and the source, provide a link to the Creative Commons licence, and indicate if changes were made. The images or other third party material in this article are included in the article's Creative Commons licence, unless indicated otherwise in a credit line to the material. If material is not included in the article's Creative Commons licence and your intended use is not permitted by statutory regulation or exceeds the permitted use, you will need to obtain permission directly from the copyright holder. To view a copy of this licence, visit http://creativecommons.org/licenses/by/4.0/. The Creative Commons Public Domain Dedication waiver (http://creativeco mmons.org/publicdomain/zero/1.0/) applies to the data made available in this article, unless otherwise stated in a credit line to the data. 
Therefore, KIT and PDGFR $\alpha$-directed tyrosine kinase inhibitors (TKIs) are widely used for advanced GISTs with activated KIT or PDGFR $\alpha$ in vitro. However, a complete pathological response is rare [3], and progression and/or secondary resistance inevitably occurs with longterm treatment of advanced disease $[4,5]$. First-line treatment with imatinib for patients with advanced GISTs results in response or tumor control in more than $80 \%$ of patients. However, nearly $50 \%$ of patients with advanced GISTs have progressive disease within two years [6]. Thus, various treatment strategies including surgery combined with TKIs, resumption of imatinib (IM), IM dose escalation or other targeting agents have been investigated to improve the survival of patients with recurrent or metastatic GISTs [7-12].

To summarize conflicting evidence comparing surgery combined with TKIs and TKIs alone in treating recurrent or metastatic GISTs, our team performed a systematic review and meta-analysis and demonstrated that surgery combined with TKIs therapy is associated with better overall survival (OS) and progression-free survival (PFS) [13]. However, the total sample size was not very large, and the certainty of evidence for both outcomes was not assessed by the Grading of Recommendations Assessment Development and Evaluations (GRADE) approach.

Over the past few years, the results of several studies concerning this topic have been published, potentially providing evidence of the effects of surgery combined with TKIs for people with recurrent or metastatic GISTs. Given the ongoing uncertainty regarding the benefits of surgery combined with TKIs, the primary objective of this systematic review is to update the previous study by identifying and incorporating recent research data to evaluate the survival benefits of surgery combined with TKIs for patients with recurrent or metastatic GISTs across all clinical settings, and the findings will be used by the Chinese Society of Clinical Oncology (CSCO) GIST guideline recommendations.

\section{Methods}

This review will update a previously published systematic review and meta-analysis by our team [13].

\section{Protocol and registration}

The present protocol has been registered at the Open Science Framework Registry (https://osf.io/xus7m). This protocol is presented in accordance with the Preferred Reporting Items for Systematic Reviews and Meta-Analysis Protocols (PRISMA-P) guidelines (see PRISMA-P checklist in Additional file 1, [14]). This updated systematic review and meta-analysis will be conducted according to the PRISMA 2020 statement and the standard methodology recommended by the Cochrane Collaboration [15-17].

\section{Inclusion criteria}

The inclusion criteria complied with the PICOS (population, intervention, comparators, outcomes and study design) description model [18] to detail the main elements. There will be no restrictions on language or publication year. The criteria that studies will be considered for inclusion are as follows:

- Population: Patients with recurrent and/or metastatic gastrointestinal stromal tumors according to the histologic examination. The study population will include all patients, with no restrictions based on country, race and ethnic origin, age, sex, or job.

- Intervention: Surgical intervention combined with TKI. Radiofrequency ablation is also regarded as a surgical intervention.

- Comparators: TKI therapy alone.

- Outcomes and measurement:

\section{Primary outcome}

- OS: death from any cause.

\section{Secondary outcome}

- PFS: progression, distant recurrence, or death from any cause.

OS and PFS are time-to-event outcomes. Thus, the hazard ratio (HR) will be used to pool overall effects [17]. HR a common assessment reported in epidemiologic studies, is defined as the hazard within the exposed groups divided by the hazard within the unexposed groups to calculate the effect of the treatment [19].

- Study design: Eligible study designs will include non-randomized studies (NRS) (case-control studies, and cohort studies) and randomized controlled trials (RCTs).

- Other inclusion criteria: Studies with a minimum of 60 months of follow-up reporting survival (time to event) outcomes. In addition, the studies need to provide sufficient data to calculate or estimate HRs and $95 \%$ confidence intervals (CIs). 


\section{Exclusion criteria}

- Studies with overlapping data.

- Conference abstracts, letters, case reports, reviews or nonclinical studies without available data will be excluded.

- There are missing or insufficient data after a reasonable attempt at contacting the corresponding authors.

- Full-text articles are not available after exhaustive searches to locate the texts.

\section{Information sources and search strategy}

A systematic search of PubMed, Embase (Ovid) and the Cochrane Central Register of Controlled Trials databases will be performed from their inceptions to $30^{\text {th }}$ December 2021 to identify all relevant studies. The details of the PubMed database search strategy are shown in Additional File 2. We will manually screen the references of all included articles to further identify additional studies meeting the eligibility criteria, and their full texts will be retrieved.

\section{Study selection and data extraction}

All study records collected in the literature search will be imported to EndNote software. After removing duplicates, two authors will independently screen all articles identified from the database search based on the eligibility criteria outlined above. First, the two independent reviewers will screen titles and abstracts. Subsequently, the two independent reviewers will reassess the full texts of the identified studies, verifying the reasons for inclusion and exclusion. The screening process will be shown in a PRISMA flow diagram [20].

Data extraction for the included studies will be conducted by two authors independently using a standardized electronic data extraction form that was piloted by all reviewers. If multiple studies are conducted on the same subjects, only the study with the highest methodological quality, the most complete results, or the most recent published date will be included [21].

All disputes in the process of study selection and data extraction will be resolved through team discussion.

\section{Dealing with missing data}

When a study does not report HR and its 95\% CI, we will contact the corresponding author of this study to request missing data via email. If no effective response is received in 14 days, we will try to estimate some or all the lnHR, the log-rank observed minus expected events (O-E), the log-rank variance and the variance of the $\ln H \mathrm{R}$ by indirect methods [22]. If even these indirect methods cannot be applied, we will estimate HRs based on Kaplan-Meier curves [22].

\section{Risk of bias assessment}

Two independent reviewers will assess the methodological quality/risk of bias of the included studies, and disagreements will be resolved by team discussion [23]. RCTs will be assessed for risk of bias with Cochrane Collaboration's tool [24]. NRS will be assessed with the Risk of Bias In Non-randomised Studies of Interventions (ROBINS-I) tool [25].

\section{Data analysis}

If identified as possible (the studies retrieved have quantitative data reported that can be combined), the extracted data will be aggregated into a meta-analysis by STATA version 15.0 (STATA, College Station, TX) software. HRs and $95 \%$ CIs will be pooled to measure time-to-event outcomes in consideration of the number and timing of events. Statistical significance will be defined as $\mathrm{p}<0.05$. HRs derived from the multivariate analysis will be used as default. When multivariate values are absent, univariate values will be used. Data from RCTs and NRS will be analysed and reported separately.

Chi-square-based Q-test will be used to check heterogeneity. The $\mathrm{I}^{2}$ test will be used to quantify the effect of heterogeneity. For chi-squared values with $\mathrm{p}<0.1$, heterogeneity will be considered to be significantly high. $\mathrm{I}^{2}$ with values $0 \%$ to $40 \%$ represents not important, $30 \%$ to $60 \%$ moderate, and $50 \%$ to $90 \%$ substantial heterogeneity, respectively [17]. Random effects models will be used for meta-analysis in cases of significant heterogeneity $\left(\mathrm{I}^{2}\right.$ $>=50 \%$ or $\mathrm{p}<0.1)$. Any comparison with high heterogeneity will be explored by subgroup analyses or sensitivity analysis. In addition, the study design and characteristics in the included studies will be analysed.

\section{A priori subgroup analyses}

If multiple studies with homogenous outcomes are reported within the following subgroups, planned subgroup analyses of the primary outcome include the following:

1) Classification of recurrent or metastatic GISTs: initially metastatic GIST versus recurrent GIST.

2) Response to preoperative TKI therapy: complete response/partial response or stable disease versus progressive disease.

3) Surgical intervention following TKI therapy: yes versus not.

4) TKI at time of surgery: imatinib treatment versus multiple lines of TKIs treatment. 
5) HR extracted from: multivariate analysis versus univariate analysis.

6) NRS with propensity score analyses : yes versus not.

\section{Sensitivity analysis}

To evaluate whether the results of the meta-analysis are substantially influenced by the presence of any individual study, we will conduct a sensitivity analysis by omitting studies with a high risk of bias.

\section{Meta-biases and quality of evidence}

If over ten studies are available, funnel plot symmetry will be used to examine publication bias [17]. We will use the GRADE approach to assess the quality of findings systematically [26], which is considered an effective method to provide detailed information on assessments [27]. The quality of findings will be classified as high, moderate, low, and very low according to four dimensions: risk of bias, inconsistency, indirectness, and imprecision. Highquality findings will indicate a high grade of confidence in intervention efficacy and quality. The GRADE assessments will be presented in a summary table.

\section{Discussion}

Our previous work was published three years ago and has some limitations [13]. Over the past years, several studies focusing on this topic have been published. This systematic review and meta-analysis update will provide a current assessment of the evidence for the role of surgery in patients with recurrent or metastatic advanced GISTs. The findings from this review will build the foundation for future research and highlight the implications for clinical practice, and the results will be used by the CSCO GIST guideline to help develop recommendations on the recurrent or metastatic disease in China.

\section{Amendments}

The protocol for the study will be amended if required. Any protocol amendments will be updated in the Open Science Framework Preregistration and explicitly described in the final manuscript.

\footnotetext{
Abbreviations

Cls: Confidence intervals; CSCO: Chinese society of clinical oncology; GRADE: Grading of Recommendations Assessment Development and Evaluations; GISTs: Gastrointestinal stromal tumors; HR: Hazard ratio; IM: Imatinib; KIT: KIT proto-oncogene, receptor tyrosine kinase; NRS: Non-randomized studies; OS: Overall survival; PDGFRa: Platelet-derived growth factor receptor alpha; PFS: Progression-free survival; PRISMA: Preferred Reporting Items for Systematic Reviews and Meta-Analyses; PRISMA-P: Preferred Reporting Items for Systematic Reviews and Meta-Analysis Protocols; RCTs: Randomized controlled trials; TKls: Tyrosine kinase inhibitors.
}

\section{Supplementary Information}

The online version contains supplementary material available at https://doi. org/10.1186/s13643-021-01863-7.

Additional file 1.

Additional file 2.

Acknowledgements

None.

\section{Authors' contributions}

Zhaolun Cai, Chunyu Xin, Zhiyuan Jiang, and Chunyu Liu provided equal contributions and shared co-first authorship.

The original idea of this research was conceived by Bo Zhang and Zhaolun Cai. Zhaolun Cai , Chunyu Xin, Zhiyuan Jiang and Chunyu Liu designed the protocol and drafted the manuscript. Xiaonan Yin, Mingchun Mu, Zhou Zhao, Chaoyong Shen, Guozhen Zhao and Yuan Yin participated in developing the eligibility criteria, search strategy, data extraction methods and data summary plan. Zhaolun Cai registered the protocol in the Open Science Framework. $\mathrm{ZB}$ and Zhaolun Cai reviewed the manuscript. All authors approved the final version of this manuscript.

Funding

This project was supported by Hainan Province Clinical Medical Center.

\section{Availability of data and materials}

Not applicable.

\section{Declarations}

\section{Ethics approval and consent to participate}

Ethical approval is not required for this protocol since the study will be conducted using previously published studies. The reporting of this protocol did not involve individual patients or public agencies.

\section{Consent for publication}

Not applicable.

\section{Competing interests}

The authors declare that they have no competing interests.

\section{Author details}

${ }^{1}$ Department of Gastrointestinal Surgery, West China Hospital, Sichuan University, Chengdu 610041, Sichuan, China. ${ }^{2}$ Institute for International Health Professions Education and Research, China Medical University, Shenyang 110122, Liaoning, China. ${ }^{3}$ Department of Pharmacy, West China Second University Hospital, Sichuan University, Chengdu 610041, Sichuan, China. ${ }^{4}$ Evidence-Based Pharmacy Center, West China Second University Hospital, Sichuan University, Chengdu 610041, Sichuan, China. ${ }^{5}$ Key Laboratory of Birth Defects and Related Diseases of Women and Children (Sichuan University), Ministry of Education, Chengdu 610041, Sichuan, China. ${ }^{6}$ West China School of Pharmacy, Sichuan University, Chengdu 610041, Sichuan, China. ${ }^{7}$ Beijing Institute of Traditional Chinese Medicine/Beijing Hospital of Traditional Chinese Medicine, Beijing 100010, China. ${ }^{8}$ Sanya People's Hospital/West China (Sanya) Hospital, Sichuan University, Sanya 572022, China.

Received: 22 January 2021 Accepted: 23 November 2021 Published online: 06 December 2021

\section{References}

1. Hirota S, Isozaki K, Moriyama Y, Hashimoto K, Nishida T, Ishiguro S, et al. Gain-of-function mutations of c-kit in human gastrointestinal stromal tumors. Science (New York, NY). 1998;279(5350):577-80.

2. Heinrich MC, Corless CL, Demetri GD, Blanke CD, von Mehren $\mathrm{M}$, Joensuu $\mathrm{H}$, et al. Kinase mutations and imatinib response in 
patients with metastatic gastrointestinal stromal tumor. J Clin Oncol. 2003;21(23):4342-9.

3. Scaife CL, Hunt KK, Patel SR, Benjamin RS, Burgess MA, Chen LL, et al. Is there a role for surgery in patients with "unresectable" CKIT+ gastrointestinal stromal tumors treated with imatinib mesylate? Am J Surg. 2003;186(6):665-9.

4. Schrage Y, Hartgrink H, Smith M, Fiore M, Rutkowski P, Tzanis D, et al. Surgical management of metastatic gastrointestinal stromal tumour. Eur $J$ Surg Oncol. 2018;44(9):1295-300.

5. Gajiwala KS, Wu JC, Christensen J, Deshmukh GD, DiehI W, DiNitto JP, et al. KIT kinase mutants show unique mechanisms of drug resistance to imatinib and sunitinib in gastrointestinal stromal tumor patients. Proc Natl Acad Sci U S A. 2009:106(5):1542-7.

6. Verweij J, Casali PG, Zalcberg J, LeCesne A, Reichardt P, Blay JY, et al. Progression-free survival in gastrointestinal stromal tumours with highdose imatinib: randomised trial. Lancet. 2004;364(9440):1127-34.

7. Comparison of two doses of imatinib for the treatment of unresectable or metastatic gastrointestinal stromal tumors: a meta-analysis of 1,640 patients. J Clin Oncol. 2010;28(7):1247-53.

8. Adenis A, Blay JY, Bui-Nguyen B, Bouche O, Bertucci F, Isambert N, et al. Masitinib in advanced gastrointestinal stromal tumor (GIST) after failure of imatinib: a randomized controlled open-label trial. Ann Oncol. 2014;25(9):1762-9.

9. Kang YK, Ryu MH, Yoo C, Ryoo BY, Kim HJ, Lee JJ, et al. Resumption of imatinib to control metastatic or unresectable gastrointestinal stromal tumours after failure of imatinib and sunitinib (RIGHT): a randomised, placebo-controlled, phase 3 trial. Lancet Oncol. 2013;14(12):1175-82.

10. Frankel TL, Chang AE, Wong SL. Surgical options for localized and advanced gastrointestinal stromal tumors. J Surg Oncol. 2011;104(8):882-7.

11. Reichardt P, Blay JY, Gelderblom H, Schlemmer M, Demetri GD, BuiNguyen B, et al. Phase III study of nilotinib versus best supportive care with or without a TKI in patients with gastrointestinal stromal tumors resistant to or intolerant of imatinib and sunitinib. Ann Oncol. 2012;23(7):1680-7.

12. Cai Z, Chen X, Zhang B, Cao D. Apatinib Treatment in Metastatic Gastrointestinal Stromal Tumor. Front Oncol. 2019;9(470).

13. Cai Z, Yin Y, Shen C, Tang S, Yin X, Chen Z, et al. Role of surgical resection for patients with recurrent or metastatic gastrointestinal stromal tumors: A systematic review and meta-analysis. Int I Surg. 2018;56:108-14.

14. Shamseer L, Moher D, Clarke M, Ghersi D, Liberati A, Petticrew M, et al. Preferred reporting items for systematic review and meta-analysis protocols (PRISMA-P) 2015: elaboration and explanation. BMJ. 2015;349:g7647.

15. Page MJ, McKenzie JE, Bossuyt PM, Boutron I, Hoffmann TC, Mulrow CD, et al. The PRISMA 2020 statement: an updated guideline for reporting systematic reviews. Syst Rev. 2021;10(1):89.

16. Cumpston M, LiT, Page MJ, Chandler J, Welch VA, Higgins JPT, et al. Updated guidance for trusted systematic reviews: a new edition of the Cochrane Handbook for Systematic Reviews of Interventions. Cochrane Database Syst Rev. 2019;10.

17. Higgins JP, Thomas J, Chandler J, Cumpston M, Li T, Page MJ, et al. Cochrane handbook for systematic reviews of interventions: Wiley; 2019.

18. Guyatt GH, Oxman AD, Kunz R, Atkins D, Brozek J, Vist GE, et al. GRADE guidelines: 2. Framing the question and deciding on important outcomes. J Clin Epidemiol. 2011:64(4):395-400.

19. Hernán MA. The Hazards of Hazard Ratios. Epidemiology. 2010;21(1):13-5.

20. Moher D, Liberati A, Tetzlaff J, Altman DG, Group P. Preferred reporting items for systematic reviews and meta-analyses: the PRISMA statement. PLoS Med. 2009;6(7):e1000097.

21. Cai Z, Liu C, Chang C, Shen C, Yin Y, Yin X, et al. Comparative safety and tolerability of approved PARP inhibitors in cancer: A systematic review and network meta-analysis. Pharmacol Res. 2021;172:105808.

22. Tierney JF, Stewart LA, Ghersi D, Burdett S, Sydes MR. Practical methods for incorporating summary time-to-event data into meta-analysis. Trials. 2007:8(1):16.

23. Jiang Z, Cai Z, Yin Y, Shen C, Huang J, Yin Y, et al. Impact of surgical margin status on the survival outcome after surgical resection of gastric cancer: a protocol for systematic review and meta-analysis. BMJ Open. 2020;10(11):e040282.
24. Higgins JP, Altman DG, Gøtzsche PC, Jüni P, Moher D, Oxman AD, et al. The Cochrane Collaboration's tool for assessing risk of bias in randomised trials. BMJ. 2011;343:d5928.

25. Sterne JA, Hernán MA, Reeves BC, Savović J, Berkman ND, Viswanathan M, et al. ROBINS-l: a tool for assessing risk of bias in non-randomised studies of interventions. BMJ. 2016;355:14919.

26. Guyatt GH, Oxman AD, Schünemann HJ, Tugwell P, Knottnerus A. GRADE guidelines: a new series of articles in the Journal of Clinical Epidemiology. J Clin Epidemiol. 2011;64(4):380-2.

27. Schunemann H, Brozek J, Guyatt G, Oxman A. Handbook for grading the quality of evidence and the strength of recommendations using the GRADE approach: The Cochrane Collaboration GRADE Working Group; 2013. gdt.gradepro.org/app/handbook/handbook.html.

\section{Publisher's Note}

Springer Nature remains neutral with regard to jurisdictional claims in published maps and institutional affiliations.

Ready to submit your research? Choose BMC and benefit from:

- fast, convenient online submission

- thorough peer review by experienced researchers in your field

- rapid publication on acceptance

- support for research data, including large and complex data types

- gold Open Access which fosters wider collaboration and increased citations

- maximum visibility for your research: over $100 \mathrm{M}$ website views per year

At BMC, research is always in progress.

Learn more biomedcentral.com/submissions 